Daimon. Revista Internacional de Filosofía, $n^{\circ} 83,2021$ pp. 139-152

ISSN: 1130-0507 (papel) y 1989-4651 (electrónico)

http://dx.doi.org/10.6018/daimon.369531

Las obras se publican en la edición electrónica de la revista bajo una licencia Creative Commons ReconocimientoNoComercial-SinObraDerivada 3.0 España (texto legal). Se pueden copiar, usar, difundir, transmitir y exponer públicamente, siempre que: i) se cite la autoría y la fuente original de su publicación (revista, editorial y URL de la obra); ii) no se usen para fines comerciales; iii) se mencione la existencia y especificaciones de esta licencia de uso. (c) (1)(-)(-)

\title{
Alimentación ontológica e incorporación ética en Levinas*
}

\section{Ontology of eating and ethics of incorporation in Levinas}

VALERIA CAMPOS SALVATERRA**

\begin{abstract}
Resumen: en el siguiente trabajo exponemos la tesis de Emmanuel Levinas según la cual en la subjetividad se entrelazan dos momentos, uno ontológico y uno propiamente ético, alejándose así del paradigma de la identidad. Sostenemos que dicha estructura co-implicada de la subjetividad puede entenderse, a partir de las dos obras principales de Levinas (Totalidad e infinito y De otro modo que ser o más allá de la esencia) a la luz de un movimiento incorporativo no-asimilativo de la alteridad. Mostramos así, en primer lugar, cómo la retórica alimentaria del comer-al y con-otro no sólo puede tener rendimiento especulativo en el caso de la tematización de la estructura ontológica del vivir de... sino que incluso funciona como catacresis fundamental en el caso de la exposición de la subjetividad como substitución. Finalmente, proponemos que estos análisis permiten una relectura de la estructura misma de la relación general con la alteridad en Levinas en términos de incorporación.
\end{abstract}

Palabras clave: Levinas, alimentación, gozo, incorporación, sustitución

\begin{abstract}
In the following work we expose Emmanuel Levinas' thesis about an altered subjectivity that has two intertwined moments: one ontological and one properly ethical. We hold that this co-implicated structure of subjectivity can be understood, in the context of the two main works of Levinas (Totality and Infinity and Otherwise tan Being or Beyond Essence), in the light of a non-assimilative incorporative movement of alterity. We show, in the first place, how the rhetoric of eating the-other and with-other has a speculative function both in the thematization of the ontological structure of the 'living of ...' and in the thesis about subjectivity as substitution - as a fundamental catachresis. Finally, we propose that these analyzes allow a re-reading of the structure of the general relationship with otherness in Levinas in terms of incorporation
\end{abstract}

Keywords: Levinas, eating, joy, incorporation, substitution

Recibido: 21/03/2019. Aceptado: 23/07/2019.

* Artículo desarrollado en el marco del proyecto FONDECYT n ${ }^{\circ} 11180180$, financiado por el Consejo Nacional de Ciencia y Tecnología de Chile.

** Profesora Auxiliar e Investigadora de la Pontificia Universidad Católica de Valparaíso. Correo electrónico: valeria.campos@pucv.cl. Líneas de investigación: ética, filosofía contemporánea, fenomenología. Libros recientemente publicados: Violencia y fenomenología. Derrida entre Husserl y Levinas (Santiago: Metales Pesados, 2017) y Transacciones peligrosas. Economías de la violencia en Jacques Derrida (Santiago: Pólvora, 2018). 
Desde sus primeros escritos, y de modo continuado tanto en Totalidad e infinito de 1961 como en De otro modo que ser o más allá de la esencia de 1974, Levinas sostiene una tesis sobre una subjetividad alterada que entrelaza un momento ético con uno ontológico ${ }^{1}$. Mientras el momento ético es el fundamento de su enfoque crítico de la tradición filosófica, especialmente en lo que concierne a las teorías del sujeto, el ontológico parece estar completamente subordinado a aquel. Sin embargo, la relación entre ambos no es completamente jerárquica, sino co-implicada, y se puede describir bajo la figura del nudo: como un nudo de la subjetivi$d a d^{2}$. En este contexto, la descripción ontológica del tema funciona al menos de dos maneras distintas, dependiendo de la época textual en la que nos movamos: ya desde De la evasión (de 1935) hasta Totalidad e infinito, el recurso efectivo a la ontología - no sólo su críticadescribe la base existencial que permite la configuración de un comando ético incondicional; o, dicho de otro modo, permite pensar una proto-dimensión del sí mismo - Levinas la llama "interioridad"3 - que posibilita la relación con la trascendencia. En De otro modo que ser..., la ontología se ocupa de señalar el punto de partida fenomenológico para operar una desestructuración de la esencia, en base a las tesis sobre el lenguaje como dualidad co-implicada Decir-Dicho (Dire-Dit), de modo de hacer que el Decir marginal al ser (le Dire d'en deça de l'être se "tematice", se "manifieste", "entre en una proposición y en un libro" $(2008,75)$.

Como puede observarse, cada caso señala una función de la ontología en la teoría ética de Levinas que es antipódico en su función explicativa respecto del otro. En Totalidad e infinito, la ontología describe una modalidad existencial en el cual la ostensión del ser - su fenomenalidad - no es producto de un proceso de temporalización definitivo, es decir, en el que el desfase de la identidad del mismo no llega aún a ser radical ${ }^{4}$. Por su parte, De otro modo que ser hace de la ontología el terreno de la esancia, un modo de la fenomenalidad como ser en el lenguaje, desde su constitución como verbo ${ }^{5}$. Lo Dicho es el modo general en que el ser puede ser descrito, en cuanto espacio de la tematización representativa (2008, 65-66). Siempre desde allí - dado que el Decir no es completamente exterior a lo Dicho, sino su margen-, Levinas intenta abrir un espacio para la aparición del Decir, modo de la expresión pre-tematizante que a su vez se enlaza con la estructura de la subjetividad como substitución: una relación cuasi-trascendental con lo otro que debe pensarse en términos de incorporación en lo mismo, impidiendo tanto su clausura como su destrucción.

1 Llamamos momento ético a la descripción de una subjetividad entendida en términos de responsabilidad, marcada afectivamente por el Deseo. Llamamos momento ontológico a la descripción de las estructuras existenciales de ese mismo sujeto, marcado afectivamente por la náusea. Cf. Campos Salvaterra, V. (2013). Náusea y Deseo: acceso afectivo a lo pre-original de la estructura subjetiva en la filosofía de Emmanuel Levinas. Pensamiento, Vol. 69, Núm. 258, pp. 7-27.

2 La tesis de una subjetividad anudada puede encontrarse ya en Totalidad e infinito, pero en De otro modo que ser o más allá de la esencia, Levinas la sostiene expresamente: " La subjectivité est précisément le noeud et le dénouement - le noeudou le dénouement - de l'essence et de l'autre de l'essence", "La subjetividad es precisamente el nudo y el desanudamiento - el nudo o el desanudamiento - de la esencia y de lo otro que la esencia" (Levinas, 2008, 23). Siguiendo la más reciente publicación de Bernasconi (2018), no existen suficientes estudios exhaustivos sobre este tema en inglés dado a confusos problemas de traducción de sus obras, lo que explicaría la reducida literatura sobre este tema en dicho idioma.

3 Cf. Levinas, 2000a, 111-202

4 Cf. Levinas, 2008, 55-60

5 Cf. al menos Levinas, 2008, 60- 61 y 67 
Estas diferencias explicativas, sin embargo, no suponen diferencias téticas estructurales. Ambas pueden, a su vez, ser enlazadas como en un nuevo nudo, ahora metodológico. Como señala Jacques Rolland, la "lógica" del otro modo (autrement) no puede describirse sino en términos de ambigüedad $(2000,148)^{6}$. O como dice Derrida en sus interpretaciones tardías sobre Levinas ${ }^{7}$, en ambos casos estamos frente a relaciones de condicionalidad que no pueden definirse por simple recurso al orden derivativo de la antero-posterioridad. Las relaciones de condicionalidad en Levinas, dice Derrida, "desafían la cronología y la lógica" $(1998,47)$. El paso de un nivel al otro se da en virtud de una nueva comprensión de la reducción fenomenológica, que opera con matices diversos en Levinas desde los comienzos de su filosofía. Se trata, como la describe en De otro modo que ser, de una reducción que no se hace "a golpe de paréntesis" (à coup de parenthèses), pero que "alimenta con su energía la interrupción ética de la esencia" $(2008,76)^{8}$.

En el presente trabajo, exploramos dos momentos de esta intriga. Por un lado, la configuración existencial que Levinas expone en Totalidad e infinito, y que describe la relación del existente con su "mundo" mediante una estructura incorporativa marcada por una fuerte retórica de la alimentación. Por otro lado, nos referiremos a ese nudo ético de la subjetividad que en De otro modo que ser es llamado substitución, y que designándose también mediante una estructura incorporativa no es descrita ya mediante el tropo del comer al otro. Queremos exponer así la siguiente tesis: que la estructura misma de la relación con la alteridad, tanto en el "seno de lo mismo" como en la trascendencia hacia el otro ser humano, sigue el movimiento de la incorporación de lo otro en lo mismo. En niveles distintos y anudados, la estructura incorporativa de la relación con la alteridad supone un proceso que se distingue de aquello que podría llamarse asimilación, que puede explicarse de modo preciso - pero catacrésico - mediante la figuración digestiva: el otro se incorpora como se incorpora la comida, pero su digestión no es completamente asimilativa y sin resto, sino que la alteridad es encriptada en el cuerpo del mismo, perturbando dicho cuerpo propio hasta alterarlo.

En Totalidad e infinito la existencia del sujeto tiene la forma intencional auto-afectiva de un "vivir de..." nutritivo y alimentario, donde lo otro de la existencia toma la forma de alimento. Pero, como dicha exterioridad no es todavía la trascendencia en sentido fuerte, el movimiento incorporativo puede ser entendido como asimilación de lo otro. En De otro modo que ser, la sustitución como alteración ética de la subjetividad implica pensar una

6 La noción de ambigüedad para caracterizar la relación entre la ontología y la ética en Levinas fue utilizada antes también por Lannoy, 1990, pp. 11-44 y posteriormente por Vanni, 2002, 108.

7 Que encontramos sobre todo expuestas en Palabra de acogida (Motd'accueill) de 1996 (1998)

8 De un modo similar, las investigaciones sobre la "posición en la existencia" del existente se realizan regresivamente, desde la experiencia de ex-cedencia o salida del ser y sus categorías que desencadena el encuentro con el otro hasta el "hacer pie en el ser" que necesariamente está supuesto en esta ex-cedencia. O como lo dice en Totalidad e infinito, dicha regresión opera como corte que "separa las relaciones análogas a la trascendencia y aquellas de la trascendencia misma" (2000a, p 112). Y aunque en ambos de estos dos últimos casos la descripción existencial del sujeto sea de fuerte inspiración heideggeriana - pues la relación del existente con su medio se da según un modo que tributa directamente a la tesis de la mostración de sí de los fenómenos-, la herencia husserliana de Levinas no deja de estar fuertemente presente, desde la crítica al anti-intelectualismo de la renovación heideggeriana de la fenomenología (cf. p.e. 2000a pp 59, 111, 128) hasta el rechazo a abandonar por completo la línea de la fenomenología constitutiva que supone mantener la reducción en el seno de una filosofía de la donación (cf. Henry, 1991). 
forma de incorporación no asimilativa. Sin embargo, a pesar de que esta estructura es incorporativa, y si bien Levinas introduce la alimentación donde Heidegger la excluye, también la excluye donde debiese introducirla: en esta relación del Mismo con lo Otro. Sin duda, lo Otro nunca puede ser asimilado por lo mismo, pero dicha interrupción de la asimilación ocurre también en los procesos digestivos, y la ambigüedad de la independencia-dependiente del existente en el movimiento del vivir de... así lo muestra. Sin embargo, dado que Levinas sí plantea en De otro modo que ser una subjetividad marcada por la necesidad de dar el pan que come, pensando así la dinámica de relación con otro bajo la forma de un comer-con él, todo indica que Levinas se negó a asumir el riesgo de pensar la relación con el otro en términos de un canibalismo especulativo; a pesar de que, de hecho, una forma especulativa y trópica de comerse-al-otro gobierna también sus tesis éticas más radicales.

En lo que sigue, expondremos ambos momentos señalados, con el fin de mostrar: 1) cómo cada momento tiene la estructura de un movimiento incorporativo no-asimilativo; 2) cómo la retórica alimentaria del comer-al y con-otro no sólo puede tener rendimiento especulativo en el caso de la tematización de la estructura del "vivir de..." sino que incluso podría funcionar como catacresis fundamental en el caso de la exposición de la subjetividad como substitución; y 3) que estos análisis permiten una relectura de la estructura misma de la relación general con la alteridad en Levinas en términos de incorporación.

\section{El Dasein no come}

En el registro de Totalidad e infinito, las tesis existenciales de Levinas están en continuidad con desarrollos tempranos que encontramos sobre todo en De la evasión de 1935 y De la existencia al existente de 1947. Entre 1940 y 1945, contamos con algunas notas de los cuadernos de cautiverio, especialmente del cuaderno número 4 (Levinas 2013, 61-101). El camino hacia la configuración de la subjetividad ontológica que se puede trazar en estos textos comienza con la tesis de la impersonalidad del ser bajo el nombre de hay (il y a $)^{9}$; sigue con los análisis de la función del presente o instante y de la posición en el ser impersonal de un existente como hipóstasis (2000b, 17); para finalizar con la tesis de la separación como modo de ser propio del existente que se realiza como vida interior o psiquismo, y cuya facultad constitutiva es la de resistir la totalización (2000a, 46). El recorrido de esta reconstrucción del sujeto está en directa relación con Heidegger y su tesis sobre la relación práctica no constituyente del Dasein con los entes intramundanos. Pero también se vincula con el recibimiento que Levinas hace de Husserl. Respecto del primero, Levinas critica la relación instrumental que constituye el estar-en-el-mundo del Dasein, principalmente en cuanto carece de apego sensible a la vida. Respecto de Husserl, se trata de tomar distancia de la tesis de la intencionalidad como relación objetivante, pero manteniéndose fiel a lo que el mismo Levinas llama "intelectualismo de la razón" (intellectualisme de la raison) (2000a, 14): referencia a un proyecto filosófico que, abrazando la posibilidad de la desmesura del pensamiento, no renuncia ni a la significación ni a la verdad.

Desde temprano Levinas afirma que la preocupación mundana del existente debe basarse en las necesidades de un cuerpo para el cual ellas no son ya penurias sino disfrute de la

9 Cf. Levinas, 1982, 1993 y 2000b, pero también 2008, 14. 
vida (Levinas, 2013, 66 $)^{10}$. Por lo tanto, construye una teoría de la existencia centrada en el concepto de gozo (jouissance) y en el movimiento transitivo del "vivir de ..." (vivre de ...). El resultado es una estructura auto-afectiva como base comprensiva de la existencia, cuya configuración en cuanto transitiva no pierde su carácter intencional, constituyendo las cosas como complementos directos y, así, en contenidos de una vida que siempre es vida-de. La vida entendida en términos de sensibilidad, gozo y alimentación se convierte en Levinas no en correlato del acto intencional de una conciencia sino es su misma condición de posibilidad (Critchley, 2004, 20). Si bien la intencionalidad en Husserl incluye a la sensación como sensación de, esta se ubica en un nivel pre-objetivo que se experimenta como una afección anterior a la aprehensión objetivadora (Herrero, 2005, 272-280). Levinas, en respuesta a esta subordinación de la afectividad, describe esta intencionalidad del gozo en términos opuestos a los planteados por Husserl: "consiste en aferrarse a la exterioridad (tenir à l'extériorité) que suspende el método trascendental incluido en la representación" (2000a, 133). Esto más que una afirmación del mundo - dice Levinas diferenciándose de Heidegger - consiste en ponerse en él corporalmente (2000a, 133).

Por esta razón, el gozo está fuertemente determinado por una retórica del comer, en la que los objetos son nutrientes para el existente y el gusto es la estructura primaria de la afectividad (2000a, 113). Las "relaciones que se producen en el seno de lo Mismo" (2000a, 112) se asemejan, dice Levinas, a las relaciones que tenemos con el alimento y, así, todo gozo es siempre alimentación (2000a, 113). En principio, esta trópica de la comida constituye al existente a través de una dinámica de incorporación de lo otro, regida por una ley de asimilación: todo lo que se incorpora toma la forma del que incorpora, reduciendo la alteridad de los alimentos a la mismidad del sujeto y constituyéndolo en esta asimilación (Levinas, 2013, 67 ${ }^{11}$. Esto le da al sujeto dominio sobre su existencia, razón por la cual Levinas habla de una "independencia del yo", que se logra como separación (séparation) de la existencia y que se garantiza con el privilegio de la alegría sobre el hambre.

Sin embargo, esta relación con los alimentos no constituye la relación trascendente que hace posible una temporalización radical de la existencia. En el proceso de síntesis temporal que el existente realiza al vivir de... las cosas no llegan a configurar ni un pasado ni un porvenir liberados de su subordinación estructural al presente (Levinas, 2000a, 153). En un primer momento de análisis, la incorporación es siempre un acto intencional auto-afectivo y, por tanto, auto-referente, que solo logra configurar el tiempo del instante sin duración (Levinas, 2000a, 155). Esta situación proto-experiencial supone, como reverso de la independencia del goce, la dependencia más pesada: aquella que en los textos tempranos se describía como la condena del yo que es siempre uno mismo (Levinas, 1982, 98), dependencia de los contenidos de una vida que no son extraños a ella misma y que la constituyen en su mismidad. La temporalización en sentido fuerte, la apertura al tiempo - al pasado inmemorial y al porvenir - y, por tanto, el más allá de la independencia en la dependencia que

10 Cuestión que ya desde La teoría de la intuición en la fenomenología de Husserl era pensada por Levinas desde una nueva y seductora forma de concebir la reducción fenomenológica: como lo que abre a la concretud misma de la existencia en cuanto vida de la conciencia, y no como un mero proceso de abstracción (2004, 186-187).

11 En el cuaderno de cautiverio $\mathrm{n}^{\circ} 4$ Levinas señala lo siguiente en relación con la necesidad: “Asimilación del mundo exterior por el sujeto. Toda necesidad satisfecha es en primer lugar saciedad, el hecho de haber comido. Primado del comer. Esta es la significación del disfrute de la necesidad". 
supone la felicidad, no se produce sino en relación con una trascendencia también radical: la del otro ser humano.

El gozo introduce así una flexión transitiva en el existir sin eliminar la flexión reflexiva (ser = ser-se) (Sucasas, 1998, 41); esto quiere decir que la identificación egológica sólo es posible a partir de la relación de carácter intencional con contenidos mundanos. Esto supone que el movimiento de interiorización al que da lugar el instante del presente como acontecimiento de nacimiento ${ }^{12}$, debe apoyarse en contenidos extra-subjetivos, en entes que, siendo trascendentes al sujeto, puedan ser reducidos en su alteridad de objetos y ser la base para la identificación eterna del sujeto. Sin embargo, esto no elimina el intervalo que aparece entre el yo y el sí mismo, pues en su transitividadel sujeto idéntico no retorna a sí inmediatamente (Levinas, 1993, 102). La existencia implica evasión y retorno: evasión hacia las cosas externas al yo, pero retorno identificante al yo de las cosas como alimento y objetos de deleite. Por ello, aun cuando hay en el gozo una fase transitiva ex-tática, él es esencialmente un movimiento de interiorización. La identificación egológica coincide con la supresión de la identidad de lo exterior; el gozo de las cosas es en sentido estricto un gozo de sí, y así se cierra el círculo inmanente del ego. La exigencia de felicidad (bonheur), propia de la naturaleza sensible del ser cuerpo, es el principio de individuación (Levinas, 2000a, 124) del existente. Sólo gozando es posible para el yo estar "en lo de sî" (chez soi): cuestión que implica a su vez vivir de lo otro; es así como el alma adquiere su identidad (Levinas, 2000a, 119).

Sin embargo, el existente que se nutre y que en dicho acto se constituye como sí mismo, no es un sujeto enteramente soberano. La noción de utensilio - el Zeug heideggeriano-, explorada principalmente por Heidegger en Ser y tiempo marca una modalidad de estaren-el-mundo según la cual el Dasein está ante todo familiarizado con una totalidad de significados ${ }^{13}$, familiarización que asegura el dominio sobre ellos en la relación de instrumentalidad. En el caso de Levinas, que las cosas sean en realidad alimentos no implica que suplan carencias, pues no debemos pensar aquí en alimentos como combustibles básicos para sobrevivir. El gozo es deleite de cosas que siempre son más que lo estrictamente necesario: "cuando se huele una flor, la finalidad del acto se limita al olor. Pasearse es tomar el aire, no con vistas a la salud, sino por el aire mismo" (Levinas, 1993, 102). Este vivir de... del gozo implica ponerse en relación con otra cosa, pero no al modo heideggeriano, pues no son originariamente instrumentos para mi ser (relación práctica-operativa con el ente intramundano), para mi cuidado ${ }^{14}$. En palabras de Francis Guibal: “A Lévinas le parece demasiado

12 Acontecimiento descrito bajo el concepto de hipóstasis. La hipóstasis es descrita en el texto del ' 47 como el paso de la existencia al existente, del verbo al sustantivo; un acontecimiento, y específicamente a un acontecimiento de nacimiento $(2000 \mathrm{~b}, 24)$. Este acontecimiento, que tiene el carácter de una conquista del ser que recomienza perpetuamente, a cada instante de la vida económica $(2000 \mathrm{~b}, 24)$. Este acontecimiento de hipóstasis es el origen de la constitución de la subjetividad ontológica como ruptura con la neutralidad del ser (Llewelyn, 1999, 43).

13 Cf. Vattimo, Gianni (1996). Introducción a Heidegger. Gedisa, Barcelona.

14 En el seminario inédito Manger l'autre dictado por Jacques Derrida en el semestre 1989-1990 en Estados Unidos y Francia, se aborda la relación entre Levinas y Heidegger respecto del comer. Dice Derrida respecto de la filosofia heideggeriana: "La chose à manger, qu'elle soit cru o cuite, carnée ou non, animale, humaine ou divine, littéralment ou figurativement, objet u non de sacrifice, me paraissait difficile à reconnaître sous le concepts de Da-sein, de Vorhandensein ou de Zuhandensein.”(Box 20, sesión 9, p. 10). El seminario se encuentra archivado en la colección especial de la biblioteca de la Universidad de California Irvine (UCI) Cf. http:// hydra.humanities.uci.edu/derrida/uci.html. 
abstracta la Sorge heideggeriana: su preocupación mundana, utilitaria e instrumental, carece de enraizamiento sensible en la vida, no remite a las necesidades carnales de un cuerpo vivo y sufrido" (2005, 65). De ahí la sentencia de Levinas: "el Dasein de Heidegger no tiene hambre jamás" (Le Dasein chez Heidegger n'a jamais faim) (2000a, 142)15.

La carencia de hambre del Dasein que nota Levinas implica para Derrida una carencia problemática de corporalidad en general (de cuerpo hambriento pero también de cuerpo sexuado) en el texto de Heidegger ${ }^{16}$, que paradójicamente hace del Dasein un ente encadenado a su existencia (Cohen, 2004, 154) ${ }^{17}$.El cuerpo ha estado presente desde los inicios de la filosofía de Levinas como elemento clave de sus análisis existenciales, siempre comprendido desde el proceso de subjetivación (Calin, 2006, 302). No habría estructura incorporativa si no fuese por el cuerpo, pues el cuerpo es él mismo la incorporación como acto consumador:

El cuerpo no es un sustantivo, en la plena acepción del término, ni la culminación de un acto - sino el acto mismo. No acto en el sentido de actividad, de empresa que se termina o continúa, sino en el sentido de cumplimiento. Algo "se [consuma"] en virtud del cuerpo (Levinas, 2013, 103).

La necesidad está dada justamente por la corporalidad propia del sujeto, es decir, está dada por una ambigüedad: la de ser libre y poder subordinar un mundo, pero estando a la vez necesitado de los contenidos de ese mundo ${ }^{18}$. Esta ambigüedad es el cuerpo (Levinas, 2000a, 119): en la subjetivación el hombre se libera de su dependencia respecto del ser impersonal, pero su cuerpo sigue siendo fuente de anhelo material. Sin embargo, esta dependencia está anudada con una independencia, ya que estas necesidades son propias de un ser capaz de asegurar la satisfacción de esas necesidades y capaz de romper la alteridad del mundo luego mediante el trabajo (Levinas, 2000a, 121); las necesidades están en mi poder, y por eso me constituyen en tanto que Mismo y no en tanto que dependiente de lo otro (Levinas, 2000a, 121). De modo paradójico, el cuerpo no es sólo un modo de esclavizarse, sino también un modo de poseer lo otro.

De lo expuesto, quisiéramos sacar la siguiente consecuencia: suficiencia y hambre, necesidad y gozo, esclavitud y dominio, y todas las demás figuras paradójicas que utiliza Levinas al referir al vivir de..., muestran que la existencia no es sino una dinámica diferencial: dependencia de la independencia y — al mismo tiempo - independencia de la dependencia. De aquí debe concluirse que la alimentación que figura el vivir de... no es una estructura simple, pues no constituye al existente ni como total soberano ni como desvalido absoluto. Por tanto, el modelo de la alimentación que explica dicha estructura no puede implicar una asimilación total del alimento, donde el sujeto que come destruye radicalmente y sin resto

15 Para una lectura que contrasta con esta ya clásica crítica a Heidegger, véase Garrido, 2012, especialmente pp. 55-63.

16 Derrida, Mangerl'autre, Box 20, sesión 9, p. 10.

17 Dice Cohen: "the very independence of subjectivity, its original separation and individuation, would lie more deeply embedded in sensibility than the synthesizing temporal ecstasies of praxis, worldliness, or anxiety, as Heidegger thought"

18 Interesante es el estudio comparativo que de Dubost $(2015,95)$ hace de Levinas y Merleau Ponty, donde el punto de distancia es exactamente esta ambigüedad dependencia-independencia que constatamos. 
su alteridad, transformándolo en sí mismo ${ }^{19}$. El alimento supera y baña al sujeto que lo muerde: en esa inmersión que el mismo realiza en su alimento, la incorporación deja de ser un movimiento cerrado y reductivo de la alteridad, y da pie para pensar un sujeto separado e independiente que, sin embargo, requiere de una ex-cedencia aún más radical. Dicha excedencia no es un situarse fuera del ser, sino la de una complejización de esta "primera" estructura de incorporación, donde la alteridad ya no puede ser asimilada, ni siquiera en términos relativos.

\section{Lo otro en lo mismo}

En De otro modo que ser Levinas no abandona del todo la figura del gozo, enfatizando su doble relación con el sí mismo que domina al alimento asimilándolo a la vez que es dominado y asimilado por él. Lo pone, en cuanto afección, en directa relación con la "sensación gustativa" (2008, 116). Saborear es allí el modo afectivo de la relación con la alteridad, más fundamental que otros modos de la percepción sensible en la medida en que la culminación o plenificación de su sentido se da en el momento de la saciedad (assouvissement). Saciedad que se refiere aquí a un momento de engullimiento (engloutissement) de lo otro, donde la distancia entre sujeto y objeto no solo queda suprimida, sino que da lugar a una total "confusión de lo sintiente y lo sentido" $(2008,117)$. En la degustación, la materia se experimenta en su no cualificación o en su no-formalización, como materia prima: llena un vacío antes de prestarse a la formalización. Es en esta descripción cómo la incorporación alimenticia y gustativa muestra al sujeto su doble relación de independencia-dependencia respecto del alimento, señalando la imposibilidad de una asimilación total por parte del sujeto; o, lo que es lo mismo, borrando el límite que separa y determina un "interior" de un "exterior"

La materia "materializa" en la saciedad que, más allá de toda referencia intencional de conocimiento y de posesión, de "apresamiento en las manos", significa el "morder sobre" irreductible al "apresamiento en las manos", pues es ya absorción por un "adentro" en la ambigüedad de dos "interioridades": aquella del "recipiente" de las formas espaciales y aquella del Yo asimilador de lo otro en su identidad enrollándose sobre sí mismo (2008, 117-118).

La pregunta es entonces quién asimila a quién. El alimento al yo o el yo al alimento. La respuesta es probablemente: ninguno a ninguno y cada uno al otro. La sensación gustativa es así, parafraseando a Levinas, un doble movimiento que hace devenir espacio al tiempo y tiempo al espacio: "el acontecimiento en el cual el fenómeno espacial de la mordedura se hace la identificación llamada yo" $(2008,118)$. La función especulativa del gozo sigue siendo aquí condicionante para la relación no totalizante con la alteridad del otro ser humano, pero también para evitar el riesgo de hacer de la pasividad de su recepción una cualidad totalmente inerte ("el anonimato de la pasividad insignificante"), de la que no emergería posibilidad alguna de significación. El gozo como auto-complacencia no completamente

19 Como lo sostiene, por ejemplo, Bernet, 2004, 87. No estamos de acuerdo con su propuesta de hacer del gozo un concepto más de la cadena que vincula poder y asimilación. 
soberana del mismo es "la condición del para-otro de la sensibilidad y de la vulnerabilidad de la exposición al otro" $(2008,119)$; pues el sentido del "dar al otro" no se obtiene sino de la posibilidad de arrancarse a sí mismo de la complacencia en el gozo, como quien "arranca el pan de su boca" para dárselo al otro: "solo un sujeto que come puede ser-para-otro o significar" $(2008,119)$.

La objetividad se construye, entonces, dando al otro lo que ya poseo, aquello de lo cual gozo. El sentido del mundo en general, lo que motiva el ejercicio gnoseológico de una conciencia-de o de una apertura existencial al ser, la mutación de la sensibilidad en intencionalidad, está dada por la significación pre-original del sentir en tanto que para-el-otro (2008, 115 y 116), lo que quiere decir que todo movimiento comprehensivo implica antes un acoger al otro hospitalariamente que luchar contra él. Esta forma general de la significación Levinas la expone progresivamente en De otro modo que ser desde la vuelta al gozo, pasando luego por la noción de proximidad (proximité) $(2008,129-155)$ que derivará en la formulación de un principio de anarquía (principe d'anarchie). Atribuida a estas alturas a una función de la huella (trace), la anarquía no es descrita ni como desorden ni caos, sino como persecución y obsesión $(2008,160)$. Pero en la medida en que se habla de un principio, la relación con lo Otro, o con la huella de lo Otro, nombra así no al otro empírico en su concretud estricta de individuo o de persona: lo nombra como principio de interrupción o de ruptura de la actividad condicionante de las estructuras trascendentales tradicionalmente atribuidas al sujeto ${ }^{20}$; principio que, en cuanto tal, tiene el estatuto de un a priori, al igual que el otorgado de jure a las condiciones estáticas que configuran lo Mismo. Sin embargo, dicho principio debe problematizarse justamente en cuanto "principio" y en cuanto "a priori". En efecto, al ser definido, manteniendo todo el rendimiento de su paradoja, como un principio de an-arquía ${ }^{21}$, Levinas señala el orden desde el cual la alteridad absoluta es posible como principio de experiencia, que no está regido ni por un ỏ@ Ǿ trascendental original, ni tampoco refiere a una materialidad desnuda. En nota al pie aclara este estatuto de principio de la anarquía: "si lo anárquico no se señalara a la conciencia reinaría a su modo. Lo anárquico no es posible más que contestado por el discurso que traiciona, pero traduce sin anularla su an-arquía por un abuso del lenguaje" $(2008,158)^{22}$. Una co-implicación, un nudo entre opuestos - principio y anarquía -, es la condición de posibilidad de toda significación, condición que no clausura ni sobre-determina dichas posibilidades, sino que las abre en la forma del para-otro.

El principio de anarquía da lugar así a una nueva tesis sobre el sujeto, a partir de la formulación lo otro en lo mismo (l'autre dans le même), que llama también substitución ${ }^{23}$. El sujeto, entendido en concordancia con la tradición como sí-mismo es, sin embargo, puesto en

20 Levinas llamará en este texto "huella" (trace) a "cierto modo de pasar inquietando el presente sin dejarse cercar

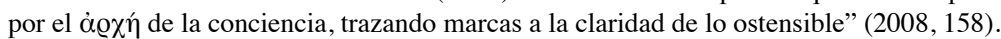

21 Cercano a lo que Reiner Schürmann propone como clave hermenéutica de un cierto heideggeriansimo deconstructivo (1982).

22 Simpatizamos con la tesis de Maloney (1997) según la cual la substitución despoja de su carácter absolutamente trascendental a la subjetividad. Sin embargo, si este gesto implica quitar de ella toda función constituyente estamos frente a un error, que señala además una escasa comprensión de la trascendentalidad husserliana.

23 Tratada in extenso en De otro modo que ser (2008), pp. 156-205. Si seguimos a Franck (2008), la substitución es la estructura fundamental que hace posible una derivación del signo como lo que sustituye a un otro y permite la significación (cf. especialmente pp. 155-160). La tesis es interesante y correcta, aunque dudamos de la función "derivativa" de la substitución de la que habla Franck. 
cuestión en su mismo movimiento de recurrencia o de retorno autoafectivo e identificante a sí, sin interrupción. Esta alteración de la subjetividad que da origen a la substitución no sería posible si la ascendencia del Otro, su advenimiento, no fuera siempre irruptivo, molesto. La an-arquía de lo Otro es obsesión, y la obsesión es persecución: "designa la forma según la cual el Yo es afectado y que es una defección (défection) de la conciencia" $(2008,160)$. La responsabilidad describe una subjetividad atenta ante la llamada del otro, pero atenta $a$ su pesar (malgré soi), porque es la persecución por el otro lo que está al fondo de la solidaridad con el otro $(2008,162)$. Para llegar al fondo de este cuestionamiento, no debemos olvidar lo que se ha tematizado respecto de una cierta configuración existencial de base formulada a partir de la noción de gozo, de experiencia gustativa, de satisfacción e incorporación, y sin que reduzcamos esta dinámica a la simple asimilación identificante. Y esto por al menos dos motivos: en primer lugar, porque la configuración del sujeto como substitución implica pensar la ipseidad como un nudo ${ }^{24}$ que entrelaza alteridad y mismidad sin reducir la una a la otra, del mismo modo que en la estructura del gozo no puede determinarse de modo certero quién se come a quién. De ahí que la obsesión, que es una acusación sin fundamento, tenga como tarea despojar al Yo de su soberbia y su imperialismo dominador. La substitución del otro por mí hace del yo un rehén (otage ${ }^{25}$. Soy rehén en la medida en que Yo ( Je) significa heme aquí (me voici), "respondiendo de todo y de todos" $(2008,180)$. Soy siempre en lugarde, ya siempre respondiendo por el otro, ese es el sentido de la responsabilidad.

En segundo lugar, la estructura del gozo y del gusto es importante porque la obsesión no es ni un acto ni un estado de conciencia, sino algo que, como la persecución, es imposible de asumir. Esta imposibilidad refiere a una pasividad absoluta (Levinas, 2008, 165) más pasiva que la pasividad de la materia, una pasividad al margen de la actividad y la pasividad, tal como entiende la tradición estas categorías (2008, 185). Por ello, Levinas describe la forma subjetiva de lo otro en lo mismo como inspiración (inspiration) (2008, 176). La subjetividad será llamada desde ahora psiquismo (psychisme) de modo de referir directamente con este dicho (Dit) al decir (Dire) que le subyace: el "sí mismo" es "lo otro en lo mismo", "donde la inspiración suscita la respiración, el pneuma mismo del psiquismo" (2008, 183). Soy así para el otro sin alienación, sino que inspirado (2008, 181), como soplo del otro que lo hace entrar en mi piel antes de todo acto voluntario. Levinas no duda en entrar en un juego trópico de sustituciones semánticas, en donde la inspiración que el otro me provoca no está lejos del acto corporal de inspiración como respiración y, así, incorporación del otro en mí que me hace su rehén; en efecto, no es sino gracias a esta trópica de la inspiración que es posible entender la condición de rehén que estructura a la subjetivad como substitución sin la carga de violencia de un secuestro o una tortura ${ }^{26}$.

24 Dice Levinas: "Presentar el nudo de la ipseidad (noeud de l'ipseité) en el hilo recto de la esencia sobre el modelo de la intencionalidad por sí, o como la apertura de la reflexión sobre sí, es poner una nueva ipseidad detrás de la ipseidad que se quisiera reducir" $(2008,166)$. Aunque, como dice bien Smith, $(2016,157)$ este nudo se manifiesta como una tautología: "el sí mismo no es ninguna otra cosa que esta substitución"

25 La palabra francesa otage comparte su raíz con la palabra hôte, que significa tanto huésped como anfitrión. Aquí ya podemos ver la relación conceptual entre el rehén y el anfitrión, relación que no se da etimológicamente en castellano. La subjetividad en tanto que hospitalidad para con el otro, es anfitriona de otro y, al mismo tiempo, también rehén.

26 Como quizás no pudo entenderla Ricoeur, calificando esta tesis de "hipérbole extrema —incluso escandalosa" (2006, 376). 
Porque estoy asignado como responsable es que soy rehén, y esta asignación tiene la forma de una abertura/apertura (overture) en el cuerpo del sí-mismo, en la que la respiración confunde actividad y pasividad $(2008,182)$. La inspiración es una abertura en la que la respiración es una modalidad que puede entenderse como ante-gusto o gusto anticipado (avant-goût) o, más exactamente, una abertura donde se "guarda el retro-gusto (arrièregoût)" (Levinas, 2008, 182). Esto quiere decir que la inspiración, como respiración pasiva que recibe al otro, es una forma del gusto como afectividad referida a la alteridad, pero que a diferencia del gusto del gozo no se sacia de ni se confunde con lo otro gustado o saboreado, sino que le es anterior; pero como en Levinas, cuestión que ya señalábamos a modo de prolegómeno, la anterioridad es siempre también posterioridad posible, ese gusto anticipado también puede leerse como una sensación que permanece después del contacto con aquello que lo causa. La inspiración guarda esta permanencia de la sensación que deja el otro al pasar por la boca, en la respiración que inspira.

Si bien Levinas habla expresamente de una cierta incorporación del otro en el mismo a través de la piel - como estar-en-su-piel (être-dans-sa-peau) o tener-al-otro-en-su-piel (avoir-l'autre-dans-sa-peau) $(2008,181)^{27}$ - , quizás no es descabellado sugerir que, antecedidas por las reflexiones sobre el gozo y el gusto, las sucesivas metáforas respiratorias hacen que Levinas termine por hacer de la boca la abertura por excelencia al otro, la condición corporal de toda abertura/apertura ${ }^{28}$. La vulnerabilidad, podemos decir, está en la boca, la boca es el orificio que nos hace más vulnerables: por donde todo entra, se cuela, se incorpora. Incluido el otro, mi prójimo, a través de su lengua que es también mi lengua, la lengua del Decir; una lengua que no es sino otra catacresis - pues Levinas ya juega con la inspiración en su doble significación - , aunque quizás la catacresis por excelencia, aquella que vincula el órgano gustativo con el lenguaje y, así, también con el otro ${ }^{29}$. Como dice Derrida en otro lugar, la boca es el lugar del aprehender en sí, del comprender, pero de un comprender que nunca es uno, y que también recibe y acoge al otro. Una boca que

[...] oralizando la cosa, nunca está lejos de comer, y el otro así comprendido se disocia difícilmente de esta instancia oral, de esta boca que comprende los labios, el paladar, los dientes, una lengua, una glotis, de esta boca que no es una, de esta boca que (sin embargo) se supone una... que come, que bebe, que tiene hambre o sed, que habla... traga, fuma, muerde, mastica, chupa, besa, saliva al otro o por el otro ${ }^{30}$.

27 Aclara Levinas ahí mismo: "Pero el psiquismo que puede significar esta alteridad en el mismo sin alienación", lo cual refiere a que la incorporación del otro no implica la destrucción total del Mismo, solo, por así decirlo, su fractura interna.

28 No olvidemos que boca tiene su antecedente en el latín os, que es la traducción del griego Jó@os, que en ambos casos señala primordialmente una abertura. Si bien estas aberturas también se sitúan en la piel, apostamos, sin embargo, por que es la boca la abertura por excelencia (¿quizás se podría decir que la piel tiene múltiples bocas?).

29 Cf., Derrida, Glas $(1974,8)$. Definiendo la catacresis en su carácter de "tropo por el cual una palabra se desvía de su sentido propio y aceptado en un lenguaje común para designar otra cosa que tiene cierta analogía con el objeto que expresa en primer lugar", Derrida da allí el siguiente ejemplo: "por ejemplo, la lengua, porque la lengua es el principal órgano de la palabra articulada".

30 Seminario Mangerl'autre, box 20, sesión 1, p. 1 
Si bien el prójimo no se come, sino que se inspira/me inspira y, al hacerlo, me da vida y me encarna ${ }^{31}$, de todas maneras estamos frente a una estructura incorporativa a la base de la afección por su alteridad. Esta estructura incorporativa, al igual que aquella referida al gozo desde Totalidad e infinito, supone la imposibilidad de pensar una asimilación total de lo que se incorpora y, como hemos señalado, un movimiento diferencial de independencia y dependencia, una co-implicación entre actividad y pasividad, soberanía y vulnerabilidad. Este modo de pensar la incorporación y de pensarla como la forma misma de la relación con la alteridad en general en un primer nivel existencial, y con la alteridad del otro ser humano en particular en el momento de la substitución, suponen una nueva vía para interpretar el estatuto general de la relación en Levinas. Citamos un pasaje que nos parece central para esta última cuestión:

Por la sustitución de los otros, el Sí-mismo escapa a la relación. En el borde de la pasividad, el Sí-mismo escapa a la pasividad o a la limitación inevitable que sufren los términos en la relación [cursivas V.C.]: en la relación incomparable de la responsabilidad, el otro no limita ya al mismo, él es soportado por aquello a lo que limita $(2008,181)$.

Sin duda y a pesar de su literalidad, esta afirmación no implica que entre el Mismo y el Otro no pueda haber relación alguna; toda una serie de tesis, en especial aquellas sobre la proximidad, así lo refutarían. Sucede, por otro lado, que es necesario para Levinas pensar la relación de otro modo, y no ya bajo la clásica forma del Mitsein, por ejemplo, "que yuxtapone los existentes dentro de un espacio común (Mitwelt) en vez de ponerlos frente a frente o, mejor dicho, cara a cara... tal compañerismo no da realmente cabida a la posibilidad de una alteridad que irrumpe y sorprende" (Guibal, 2005, 65). Aun cuando la intersubjetividad del Mitsein sea tan constitutiva del Dasein como el estar-en-el-mundo, esta manera de entenderla no "abre verdaderamente una brecha en el espacio ensimismado de un Dasein que sigue ubicándose en el centro del mundo, sin interlocutores ni interpelación que puedan descentrarlo..." (Guibal, 2005, 65). La contigüidad del con debe ser re-pensada, cuestionada y reformulada según la estructura de lo otro en lo mismo: según una estructura incorporativa. En efecto, que lo otro esté en lo mismo permite a Levinas no abandonar radicalmente una teoría del sujeto, al tiempo que criticarla. En una línea cercana a la de Derrida, que en una entrevista de 1989 afirma que no se puede realmente "liquidar" ("liquider") al sujeto, sino someterlo a un ejercicio crítico de desmontaje, tachadura o desconstrucción de sus supuestos metafísicos (Derrida, 1992, 270). Y si, por otro lado, sostenemos con Derrida también que describir la dislocación del sujeto absoluto desde el otro y desde el tiempo, como hace Levinas, "no conduce más allá de la fenomenología" (Derrida, 1992, 278), no se trata, por tanto, de destruir el sí-mismo, la ipseidad como punto de partida y llegada de la experiencia significativa. Como señala Levinas, para que esta ipseidad pueda operar en una nueva teoría

31 Son muchos los lugares del texto del '74 (2008) donde Levinas se refiere a la encarnación necesaria del Mismo para recibir al otro, encarnación que es al tiempo solo posibilitada por la llegada del otro: 114, 124, 125, 127. En este último lugar, Levinas dice: "La identidad aquí se hace no por confirmación de sí, sino, significación del uno-para-el-otro, por deposición (déposition) de sí, deposición que es la encarnación del sujeto o la posibilidad misma de dar, de ceder significancia (baillersignifiance)" 
del sujeto debe pensarse como un Yo que es un otro (Je est un autre), "fuera de todo lugar, en sí - al margen de la autonomía de la auto-afección y de la identidad que repose sobre ella misma" (Levinas, 2008, 187). Esta relación de estructura incorporativa no hace del sujeto más que una "experiencia finita de la no-identidad a sí, de la interpelación inderivable en tanto viene del otro, de la huella del otro" (Derrida, 2005, 9). Levinas dice hacia el final de De otro modo que ser que este libro ha mostrado que el uno como rehén del otro revela su pasividad de sujeto en tanto trasciende la esencia comprendida como poder y acto: "en su identidad de invocado irremplazable, sin retorno a sî" $(2008,221)$, de modo que la substitución no es sino una "denucleación del núcleo substancial del Yo que se forma en el Mismo, fisión del núcleo 'misterioso' de la 'interioridad' del sujeto" (2008, 221). No es necesario ir demasiado lejos hasta forzar en el texto levinasiano el desplazamiento trópico hacia una figuración alimentaria que esta estructura incorporativa supone, pues se trata de pensar una des-igualdad en el sí mismo dada por substitución, que es descrita también como un "sí desfasado por sí, olvidado de sí, olvidado en la mordedura sobre sí (morsure sur soi) [cursivas V.C.], en la referencia a sí por el devorarse del remordimiento (le se ronger du remords)" $(2008,182)$.

\section{Bibliografía}

Bernasconi, R. (2018). “'Subjectivity Must Be Defended’: Substitution, Entanglement, and the Prehistory of the Me in Levinas". Morgan, M. (Ed.), The Oxford Handbook of Levinas. Oxford: Oxford University Press.

Bernet, R. (2004). "Levinas' critique of Husserl”. En Critchley, S. \& Bernasconi, R. (Eds.), The Cambridge Companion to Levinas. Cambridge: Cambridge University Press.

Calin, R. (2006). "Le corps de la responsabilité. Sensibilité, corporéité et subjectivité chez Levinas". Les études philosophiques, vol 3, n 78, pp. 279-318.

Campos Salvaterra, V. (2013). "Náusea y Deseo: acceso afectivo a lo pre-original de la estructura subjetiva en la filosofía de Emmanuel Levinas". Pensamiento, Vol. 69, Núm. 258, pp. 7-27.

Critchley, S. (2004) "Introduction”, in Critchley, S. \& Bernasconi, R. (Eds.), The Cambridge Companionto Levinas. Cambridge: CambirdgeUniversityPress.

Cohen, R. (2004). Ethics, Exegesis and Philosophy. Interpretation After Levinas. Cambridge: Cambridge University Press.

Derrida, J. (1967). De la grammatologie. París: Minuit. Edición castellana de 2005, México: Siglo XXI. Traducción de Oscar del Barco y Conrado Ceretti.

Derrida, J. (1974). Glas. Paris: Galilée.

Derrida, J. (1989-1990). Mangerl'autre: politiques de l'amitié. Seminario inédito archivado en la Derrida Collection / Critical Theory Collection / Special Collections and Archives/ UCI Library, California, USA. Box 10, files 8-15.

Derrida, J. (1992). “'Il faut bien manger' ou le calcul du sujet”. En Points de suspensión. Entretiens, 269-302. Paris: Galilée.

Derrida, J. (1998). "Palabra de acogida". En Adiós a Emmanuel Levinas. Traducción de Julián Santos. Madrid: Trotta.

Dubost, M. (2015). "La jouissance selon Levinas Une lecture critique de l' «empiètement de chair» de Merleau-Ponty”. Revue Philosophique de Louvain113(1), 85-104. 
Farrell, D. (2006). All You Can't Eat: Derrida's Course, “Rhétorique Du Cannibalisme” (1990-1991). Research in Phenomenology, Vol. 36 (2006), pp. 130-180

Franck, D. (2008). L'un-pour-l'autre. Levinas et la signification. Paris: PUF.

Garrido, J.M. (2012).On Time, Being and Hunger. Challenging the Traditional Way of Thinking Life. New York: Fordham University Press.

Goldstein, D. (2010). "Levinas'ontology of eating”. En Gastronomica, Vol. 10, No. 3 (Summer 2010), pp. 34-44. Published by: University of California Press

Guibal, F. (2005). Fenomenología, ontología, metafísica. Santiago: Colección Teoría.

Henry, M. (1991). "Quatreprincipes de la phénoménologie”, en Revue de métaphysique et de morale, $1 / 1991$.

Herrero, F. J. (2005). De Husserl a Lévinas. Un camino en la fenomenología. Salamanca: Universidad Pontificia de Salamanca.

Lanoy, J. L. (1990) “D’une ambigüité”, en Etudes phénoménologiques, nº 12.

Levinas, E. (1982). De l'évasion. Paris: Fata Morgana.

Levinas, E. (1993). El tiempo y el otro. Traducción de José Luis Pardo. Barcelona: Paidós.

Levinas, E. (2000a) Totalité et infini. Essais sur l'extériorité. Paris: Livre de Poche.

Levinas, E. (2000b). De la existencia al existente. Traducción de Patricio Peñalver. Madrid: Arena.

Levinas, E. (2004). La teoría fenomenológica de la intuición. Traducción de Tania Checci. Salamanca: Sígueme.

Levinas, E. (2008). Autrement qu'être ou au-delà de l'essence. Paris: Livre de Poche.

Levinas, E. (2013). Escritos inéditos 1. Traducción de Miguel García Baró. Madrid: Trotta Llewelyn, J. (1999). La genealogía de la ética. Madrid: Encuentro.

Maloney, P. (1997). "Levinas, substitution, and transcendental subjectivity". Man and World 30: 49-64.

Ricoeur, P. (2006). Sí mismo como otro. Traducción de Agustín Neira Castro. México: Siglo XXI.

Rolland, J. (2000) Parcours de l'autrement. Lectured'EmmanuelLévinas. Paris: Pressesuniversitaires de France.

Smith, D. (2016). “'Afteryou, sir!': Substitution in Kant and Levinas”. Journal of the British SocietyforPhenomenology, 48:2, 149-161.

Sucasas, J.A. (1998) La subjetivación. Hipóstasis y gozo, en Anthropos $\mathrm{n}^{\mathrm{o}} 176$ enero-febrero, 38-43.

Vanni, M. (2002). “Transcendance et ambiguïté. Quelques problèmes d'interprétation de la pensée de Lévinas”. En Les Études philosophiques 2002/1 (n 60), 103-122.

Vattimo, G. (1996). Introducción a Heidegger. Barcelona: Gedisa. 